\title{
Theory of Mass Transfer from a Sphere and a Circular \\ Cylinder in Pulsating Flow*
}

\section{By Munekazu OHMI** and Tateo USUI**}

\section{Synopsis}

Generalized expressions for both the local and the overall mass-transfer from a sphere and a circular cylinder in steady, oscillating, and pulsating flows are derived by using solutions to velocities around the bodies. The integrals included in these expressions being evaluated numerically, the local mass-transfer distribution profiles around the bodies and the overall masstransfer expressions are given for these cases.

Comparison between local mass-transfer distributions around the bodies in steady and pulsating flows shows that pulsating flow accelerates the mass transfer particularly on the rear side of the body, where the mass transfer under steady flow is small because of the dead-water region. The approximate expressions of overall mass-transfer under pulsating flow are

$$
S h=2+\left[(0.654)^{1.85}+\left(0.648 z^{1 / 3}\right)^{1.85}\right]^{1 / 1.85} S c^{1 / 3} \operatorname{Re}_{p}^{1 / 2}
$$

for a sphere and

$$
S h=\left[(0.615)^{1.74}+\left(0.728 z^{1 / 3}\right)^{1.74}\right]^{1 / 1.74} S c^{1 / 3} \operatorname{Re}_{p}^{1 / 2}
$$

for a circular cylinder, where

and

$$
S h=2 r_{o} k_{f} / D, \quad z=\left(a \omega / U_{\infty}\right)^{3 / 2}\left(a / r_{o}\right)^{1 / 2}, \quad S c=\nu / D
$$

$$
R e_{p}=2 r_{o} U_{\infty} / \nu
$$

(a: amplitude, $D:$ diffusivity, $k_{f}:$ mass-transfer coefficient, $r_{o}:$ radius, $U_{\infty}:$ free stream velocity, $\nu:$ kinematic viscosity, $\omega:$ angular frequency).

These expressions coincide with ones under steady flow, i.e.,

$$
\begin{aligned}
& S h=2+0.654 S c^{1 / 3} R_{p}^{1 / 2} \quad \text { (sphere), } \\
& S h=0.615 S c^{1 / 3} R_{p}^{1 / 2} \quad \text { (circular cylinder) }
\end{aligned}
$$

and ones under oscillating flow, i.e.,

$$
\begin{aligned}
& \left.S h=2+0.917 S c^{1 / 3}\left(r_{o} a \omega / \nu\right)^{1 / 2}\left(a / r_{o}\right)^{1 / 6} \quad \text { (sphere }\right), \\
& S h=1.03 S c^{1 / 3}\left(r_{o} a \omega / \nu\right)^{1 / 2}\left(a / r_{o}\right)^{1 / 6} \quad \text { (circular cylinder), }
\end{aligned}
$$

according as $z \rightarrow 0$ and $z \rightarrow \infty$, respectively. These results are compared with analytical and experimental ones reported previously, and shown to be rather satisfactory.

\section{Introduction}

In a preceding paper, ${ }^{1)}$ an acceleration effect of pulsating flow of hydrogen on the rate of reduction of single iron oxide pellets was examined theoretically by the use of the unreacted-core shrinking model for one interface. ${ }^{2}$ It was assumed that the pulsating flow affected neither the rate constant of chemical reaction nor the intraparticle effective diffusivity, but increased the mass-transfer coefficient in the gas film, $k_{f}$, which was evaluated by an expression given by Kitaura et al. ${ }^{3)}$ However, partly because this expression was a semi-empirical one obtained from both theoretical and experimental analyses of mass transfer around an oscillating sphere in steady flow, partly because the range of flow conditions such as amplitude, frequency, and flow rate over which the calculations were carried out ${ }^{1)}$ was wider than that of their experiments, ${ }^{3)}$ re-examination of their expression has been considered necessary.

There are some reports on the mass-transfer from a sphere and a circular cylinder oscillating in a stagnant fluid. Noordsij and Rotte ${ }^{4)}$ gave an empirical equation of the overall mass-transfer coefficient for a sphere. Jameson ${ }^{5)}$ derived the overall coefficient for a circular cylinder, while Sugano and Ratkowsky ${ }^{6}$ ) gave an empirical equation. However, there appears to be no work except Kitaura et al.'s on the overall mass-transfer coefficient for these bodies in pulsating flow or for those oscillating in steady flow.

The purpose of this paper is to present a theory of mass transfer from a sphere (S.) and a circular cylinder (C.C.) in steady and pulsating flows; the local and the overall mass-transfer coefficients are analytically derived. Moreover, the acceleration effect of pulsation upon the mass-transfer rate is discussed.

\section{Analysis}

\section{Basic Equations}

In mass transfer from these bodies to a fluid, the analysis is restricted to the region very close to them, where the velocity component $v$ is negligibly small; the term $v \partial C / \partial y$ in the equation for mass transfer is negligible. Therefore, the equation in both cases of steady flow and unsteady flow in which a quasisteady-state assumption can be made (that is, $S c \gg 1)^{5}$ ) is approximated as

$$
u_{t a} \partial C / \partial x=D \partial^{2} C / \partial y^{2}
$$

with boundary conditions

$$
x=0 \text { or } y=\infty: C=0, \quad y=0: \quad C=C_{0} .
$$

By inserting the relation $x=r_{0} \theta$ into Eq. (1) and carrying out a mass balance along a streamline of value $\psi$, an approximately equivalent form of $\mathrm{Eq}$. (1) is derived $^{\text {7) }}$ as follows:

$$
u_{t a}(\partial C / \partial \theta)_{\psi}=r_{o} D\left(\partial^{2} C / \partial y^{2}\right)_{\theta}
$$

Now the unequality $S c \gg 1$ is also assumed in the case of steady flow. Then, because the present analysis is restricted to a system where the diffusional boundary layer is considerably thinner than the velocity boundary layer, the time-averaged velocity gradient in the layer very close to each body may be assumed constant:

$$
u_{t a}=\beta y, \beta=\left(\partial u_{t a} / \partial y\right)_{y=0} .
$$

* Originally published in Tetsu-to-Hagané, 63 (1977), 1633, in Japanese. English version received June 25, 1981. CC 1982 ISIJ

** Department of Metallurgical Engineering, Faculty of Engineering, Osaka University, Yamadaoka, Suita 565. 
Therefore, the stream function for a sphere is evaluated as

$$
\psi=\int_{0}^{y} u_{t a} r d y=\frac{1}{2} \beta r_{o} y^{2} \sin \theta
$$

The concentration gradient at the wall can be derived from Eqs. (2) to (5) as follows:*

$$
\left(\frac{\partial C}{\partial y}\right)_{y=0}=-\frac{C_{o}}{\Gamma(1 / 3) \Delta}\left(\frac{3}{\phi D}\right)^{1 / 3}
$$

where,

$$
\begin{aligned}
& d \phi / d \theta=r_{o} /\left(\beta \Delta^{3}\right), \ldots \ldots \\
& \Delta=\sqrt{2 \psi_{\Delta} /\left(\beta r_{o} \sin \theta\right)}
\end{aligned}
$$$$
\text { (S.), }
$$

and $\phi_{\Delta}$ is the stream function at $y=\Delta, \Delta$ the thickness of the imagined boundary layer in which all the mass transfer occurs. From Eqs. (6) to (8) and (8-a), the local mass-transfer coefficients are given by

$$
\begin{aligned}
& k_{f \theta}=-\left(D / C_{o}\right)(\partial C / \partial y)_{y=0} \\
& =\left\{\begin{array}{l}
\frac{3^{1 / 3}}{\Gamma(1 / 3)} \frac{D^{2 / 3}}{r_{o}^{1 / 3}}[\beta \sin \theta]^{1 / 2}\left[\int_{0}^{\theta} \beta^{1 / 2} \sin ^{3 / 2} \theta d \theta\right]^{-1 / 3} \\
\frac{3^{1 / 3}}{\Gamma(1 / 3)} \frac{D^{2 / 3}}{r_{o}^{1 / 3}} \beta^{1 / 2}\left[\int_{0}^{\theta} \beta^{1 / 2} d \theta\right]^{-1 / 3} \quad \text { (C. C. .). }
\end{array}\right.
\end{aligned}
$$

\section{Mass Transfer in Steady Flow}

Solutions to the velocities around a sphere ${ }^{8,9)}$ and a circular cylinder ${ }^{8)}$ in steady flow having a free stream velocity $U_{\infty}$ have been derived from the boundary-layer theory; $\beta$ in Eq. (4) is written as

$$
\beta=\sqrt{U_{\infty}^{3} /\left(r_{0} \nu\right)} F(\theta),
$$

where,

$$
F(\theta)=\left\{\begin{aligned}
f_{s}(\theta)= & \sqrt{3}\left(1.3916 \theta-0.5402 \theta^{3}\right. \\
& \left.+0.0585 \theta^{5}-0.00363 \theta^{7}+\cdots\right) \quad \text { (S.) } \\
f_{c}(\theta)= & \sqrt{2}\left(2.4652 \theta-0.9659 \theta^{3}+0.1032 \theta^{5}\right. \\
& -0.00647 \theta^{7}+0.0000154 \theta^{9} \\
- & 0.0000407 \theta^{11}+\cdots
\end{aligned}\right.
$$

Then, combination of Eqs. (9) and (10) yields the following local Sherwood number:

$$
S h_{\theta}=\left[2^{1 / 2} 3^{1 / 3} / \Gamma(1 / 3)\right] G(\theta) S c^{1 / 3} R e_{p}^{1 / 2}
$$

and the overall Sherwood number is, therefore, given by

$$
S h=\left[2^{1 / 2} 3^{1 / 3} / \Gamma(1 / 3)\right]\left[I\left(\theta_{1}\right) / J\left(\theta_{2}\right)\right] S c^{1 / 3} R e_{p}^{1 / 2}, \ldots
$$
where,

$$
G(\theta)=\left\{\begin{array}{l}
{[F(\theta) \sin \theta]^{1 / 2}\left[\int_{0}^{\theta} F(\theta)^{1 / 2} \sin ^{3 / 2} \theta d \theta\right]^{-1 / 3}} \\
F(\theta)^{1 / 2}\left[\int_{0}^{\theta} F(\theta)^{1 / 2} d \theta\right]^{-1 / 3}
\end{array}\right.
$$

$$
\begin{aligned}
I\left(\theta_{1}\right) & = \begin{cases}\int_{0}^{\theta_{1}} G(\theta) \sin \theta d \theta & \text { (S.) } \\
\int_{0}^{\theta_{1}} G(\theta) d \theta & \text { (C. C. }),\end{cases} \\
J\left(\theta_{2}\right) & = \begin{cases}\int_{0}^{\theta_{2}} \sin \theta d \theta & \text { (S.) } \\
\theta_{2} & \text { (C. C.), }\end{cases}
\end{aligned}
$$

and

$$
\theta_{2}=\pi, \quad \theta_{1}=\theta_{s}= \begin{cases}(109.6 / 180) \pi & \text { (S.) } \\ (108.8 / 180) \pi & \text { (C. C. }) .\end{cases}
$$

Here, the upper limit of the integration range in Eq. (15), $\theta_{1}$, is set equal to $\theta_{s}$, because the region from the separation point $\theta_{s}$ to $\theta=\pi$ is assumed to be the deadwater region in which the fluid is stagnant.

\section{Mass Transfer in Oscillating Flow}

Analyses for flows around the bodies immersed in a fluid oscillating harmonically with small amplitude are replaced by analyses for flows around the bodies oscillating harmonically with small amplitude in a stagnant fluid. To be exact, these two kinds of analyses will be different, but such a replacement may be approximately applicable, ${ }^{10}$ ) because the fluid dealt with in this paper is incompressible.

Then, from the analyses of Appendix (see Eqs. (A3), (A-5), (A-16), (A-23), and (A-25)) and Schlichting, ${ }^{11)} \beta$ in Eq. (4) is written as

$$
\beta=\left(a^{2} / r_{o}\right) \sqrt{\omega^{3} / \nu} F(\theta),
$$

where,

$$
F(\theta)= \begin{cases}\left(3^{2} / 2^{9 / 2}\right) \sin 2 \theta & \text { (S.) } \\ (1 / \sqrt{ } 2) \sin 2 \theta & \text { (C. C. }) .\end{cases}
$$

Insertion of Eq. (18) into Eq. (9) yields the following local Sherwood number:

$$
S h_{\theta}=\left[2 \cdot 3^{1 / 3} / \Gamma(1 / 3)\right] G(\theta) S c^{1 / 3}\left(r_{o} a \omega / \nu\right)^{1 / 2}\left(a / r_{o}\right)^{1 / 6} \ldots
$$

and hence the overall Sherwood number is given by

$$
\begin{aligned}
S h= & {\left[2 \cdot 3^{1 / 3} / \Gamma(1 / 3)\right]\left[I\left(\theta_{1}\right) / J\left(\theta_{2}\right)\right] S c^{1 / 3} } \\
& \times\left(r_{o} a \omega / \nu\right)^{1 / 2}\left(a / r_{o}\right)^{1 / 6}, \quad \ldots \ldots \ldots \ldots . . .
\end{aligned}
$$

where $G(\theta), I\left(\theta_{1}\right)$, and $J\left(\theta_{2}\right)$ are already defined as Eqs. (14) to (16). Because oscillating flows around a sphere and a circular cylinder are symmetrical about $\theta=\pi / 2$, it can be taken that

$$
\left.\theta_{1}=\theta_{2}=\pi / 2 \quad \text { (S. and C. C. }\right) \text {. }
$$

\section{Mass Transfer in Pulsating Flow}

Pulsating flow field can be approximately represented by superposing steady flow field upon oscillating flow field. ${ }^{12,13)}$ Hence, from Eqs. (10), (11), (18) and (19) $\beta$ can be written as

* For a circular cylinder, Jameson ${ }^{5}$ ) derived the same solution as Eq. (6) with Eq. (7) and the following equations:

$$
\begin{aligned}
& \phi=\int_{0}^{y} u_{t a} d y=\frac{1}{2} \beta y^{2} \\
& \Delta=\sqrt{2 \psi_{\Delta} / \beta}
\end{aligned}
$$




$$
\beta=\left\{\begin{array}{l}
\sqrt{U_{\infty}^{3} /\left(r_{o} \nu\right)} f_{s}(\theta) \\
\quad+\left(a^{2} / r_{o}\right) \sqrt{\omega^{3} / \nu}\left(3^{2} / 2^{9 / 2}\right) \sin 2 \theta \\
\sqrt{U_{\infty}^{3} /\left(r_{o} \nu\right) f_{c}(\theta)} \\
\quad+\left(a^{2} / r_{o}\right) \sqrt{\omega^{3} / \nu}(1 / \sqrt{2}) \sin 2 \theta
\end{array}\right.
$$

These are reduced to Eq. (10), in which

$$
\begin{aligned}
F(\theta) & = \begin{cases}f_{s}(\theta)+\left(3^{2} / 2^{9 / 2}\right) z \sin 2 \theta & \text { (S.) } \\
f_{c}(\theta)+(z / \sqrt{2}) \sin 2 \theta & \text { (C. C. }),\end{cases} \\
z & =\left(a \omega / U_{\infty}\right)^{3 / 2}\left(a / r_{o}\right)^{1 / 2} .
\end{aligned}
$$

Then, from Eqs. (9) and (23) or (10), the local and overall Sherwood numbers are given by Eqs. (12) to (16) and

$$
\theta_{1}=\theta_{2}=\pi \quad \text { (S. and C. C.). }
$$

Because there is a point $\theta=\theta_{s}^{\prime}$ (time-averaged separation point) at which $\beta=0$ in Eq. (23), the integration ranges in Eqs. (14) and (15) have to be divided into the following three parts:

$$
0 \leqq \theta \leqq \theta_{s}^{\prime}, \quad \theta_{s}^{\prime} \leqq \theta \leqq \theta_{s}, \text { and } \theta_{s} \leqq \theta \leqq \pi .
$$

The parameter $\left(a \omega / U_{\infty}\right)$ in the dimensionless variable $z$ is the ratio of a characteristic velocity of oscillating component, $a \omega$, and that of steady component, $U_{\infty}$, and the other $\left(a / r_{o}\right)$ is dimensionless amplitude. Kitaura et al. ${ }^{3)}$ derived two terms $\left(a \omega / U_{\infty}\right)^{2}$ and $\sqrt{U_{\infty} /\left(r_{o} \omega\right)}$ from theoretical considerations as terms proportional to the ratio of oscillating pressure to viscous force and proportional to the dimensionless boundary layer thickness, respectively, and introduced the product of the two into semi-empirical correlation equation; the product is just the same as $z$.

By the way, in the case of a sphere, if the fluid is at rest, the Sherwood number is $S h=2 .{ }^{14)} \quad$ Because this fact is independent of any forced convection, the Sherwood number for a certain convection has to be added by this value.

\section{Results and Discussions}

Both the local and the overall mass-transfer coefficients for a sphere and a circular cylinder in steady, oscillating, and pulsating flows are calculated numerically from the analytical results shown in the previous section. Integrations in Eqs. (14) and (15) are carried out by the use of Simpson's rule with angle interval of $0.5 \mathrm{deg}$. For example, the coefficient (1.03) in Eq. (32) is given, in detail, as 1.033 by the present calculation and as 1.032 by the calculation using Beta function. ${ }^{5,6)}$ The accuracy of the present calculations are, therefore, considered good enough.

\section{Local Variation of Mass Transfer}

Figure 1 exemplifies mass-transfer distribution profiles around a sphere in steady, oscillating, and pulsating flows. The local Sherwood numbers $S h_{\theta}$ for steady, oscillating, and pulsating flows have the same minimum value $S h_{\theta}=2$ at $\theta=\theta_{s}, 90 \mathrm{deg}$, and $\theta_{s}^{\prime}$, respectively, where $\theta_{s}^{\prime}$ depends on $z$ and $90 \mathrm{deg}<\theta_{s}^{\prime}<$ $\theta_{s}=109.6 \mathrm{deg}$. Comparison of these distributions

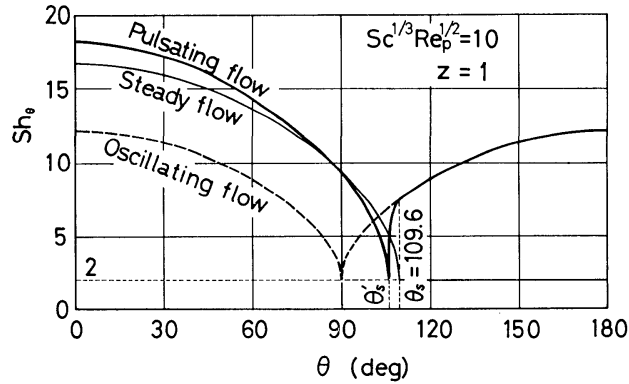

Fig. 1. Local variations of mass transfer around a sphere under steady, oscillating, and pulsating flows.

shows that pulsating flow accelerates the mass transfer particularly on the rear side of the body, where the mass transfer under steady flow is small because of the dead-water region. Local variation of mass transfer around a circular cylinder has the same tendency as that around a sphere, except that the stagnation point under steady flow is at $\theta_{s}=108.8 \mathrm{deg}$ and the minimum value is $S h_{\theta}=0$.

Such local variations of mass transfer have been measured under steady flow by Frössling, ${ }^{15}$ ) Linton and Sutherland, ${ }^{16)}$ and Gibert and Angelino ${ }^{17}$ ) (for a sphere) and by Lewis ${ }^{18)}$ and Schlichting ${ }^{8)}$ (for a circular cylinder), and under pulsating flow by Lebouche and Martin $^{19)}$ (for a circular cylinder), and agree qualitatively with Fig. 1. However, in case of steady flow, those experimental results show that the flow in the dead-water region increases gradually as the Reynolds number $R e_{p}$ rises, and local Sherwood numbers for a sphere and a circular cylinder become greater than $S h_{\theta}=2$ and 0 , respectively. Such a tendency is found in the distribution profiles obtained from the numerical analyses for a sphere by Grafton ${ }^{20)}$ and Ihme et al. ${ }^{21)}$ The present results represent neither the phenomena mentioned above nor that the separation point or the minimum mass-transfer point moves a little towards the front stagnation point as $R e_{p}$ increases. Therefore, the present analysis is, in a rule, restricted within a range of relatively low Reynolds numbers.

\section{Overall Mass-transfer \\ 1. Steady Flow}

From Eqs. (11), (13) to (17), the resultant equations for overall mass-transfer under steady flow are

$$
S h= \begin{cases}2+0.654 S c^{1 / 3} R e_{p}^{1 / 2} & \text { (S.) }, \ldots \\ 0.615 S c^{1 / 3} R e_{p}^{1 / 2} & \text { (C. C. }) .\end{cases}
$$

In a previous paper, ${ }^{2)}$ values of $A$ in the following equation reported formerly for a sphere:

$$
S h=2+A S c^{1 / 3} R e_{p}^{1 / 2}
$$

were correlated with the Reynolds number range in which experiments were carried out, and shown to have a tendency to increase slightly as the Reynolds number rises, but it was also shown to be appropriate to use $A=0.55$ within the Reynolds number range $0.1 \leqq R e_{p} \leqq 2000$. Equation (28) derived here has just the same functional-form as Eq. (30) and nearly 
the same coefficient-value. The amount of mass transfer is considered to be evaluated lower than the appropriate value, because it is assumed that the fluid in the dead water region is at rest and the velocity component $v$ is negligible. However, this coefficient value $(0.654)$ is, in fact, larger than those reported previously. It is, therefore, necessary to re-examine the coefficient value by experiments, and that is reported elsewhere. ${ }^{13)}$

Table 1 shows theoretical and experimental results reported previously for a circular cylinder; this approximate equation is considered satisfactory.

\section{Oscillating Flow}

From Eqs. (14) to (16), (19), (21), and (22), numerically calculated equations for overall mass-transfer under oscillating flow are

$$
S h= \begin{cases}2+0.917 S c^{1 / 3}\left(r_{o} a \omega / \nu\right)^{1 / 2}\left(a / r_{o}\right)^{1 / 6} & \text { (S. }), \ldots \ldots(31) \\ 1.03 S c^{1 / 3}\left(r_{o} a \omega / \nu\right)^{1 / 2}\left(a / r_{o}\right)^{1 / 6} & (\text { C. C. }) .{ }^{5,6)}\end{cases}
$$

Now, comparison is made between Eq. (31) and (semi-)empirical correlations reported previously under conditions that natural convection is negligibly small, namely, frequency and/or amplitude of oscillation are comparatively large. The correlations of mass transfer from a sphere are summarized in Table 2 , by using the following equation:

$$
S h=B_{1}+B_{2} S c^{1 / 3}\left(r_{o} a \omega / \nu\right)^{1 / 2} .
$$

Comparison of Eq. (31) with these results shows that the functional form of Eq. (31) is relatively exact and the coefficient value in the term $B_{2}, 0.917$, rather satisfactory. However, it is necessary to examine the correlation equation by experiments, because there have been only a few reports on this subject.

\section{Pulsating Flow}

It is understood from Eqs. (13) to (16) and (24) to (27) that the Sherwood numbers for a sphere and a circular cylinder in pulsating flow are certain functions of $z$; these relations are shown schematically in Fig. 2. Each curve has two asymptotes, Eq. (28) or (29) as $z \rightarrow 0$, and Eq. (31) or (32) as $z \rightarrow \infty$. Thus, the following equations

$$
S h=\left\{\begin{array}{r}
2+\left[(0.654)^{\kappa}+\left(0.917 z^{1 / 3} / \sqrt{ } 2\right)^{\kappa}\right]^{1 / \kappa} S c^{1 / 3} R e_{p}^{1 / 2} \\
\text { (S.) } \ldots \ldots \ldots(3 \\
{\left[(0.615)^{\kappa}+\left(1.03 z^{1 / 3} / \sqrt{ } 2\right)^{\kappa}\right]^{1 / \kappa} S c^{1 / 3} R e_{p}^{1 / 2}} \\
\text { (C. C. }) \ldots \ldots(3
\end{array}\right.
$$

are tentatively made as approximate equations, and the optimum values for $\kappa$ are examined, as shown in Fig. 3. Then, by choosing $\kappa=1.85$ for a sphere and $\kappa=1.74$ for a circular cylinder, these equations approximate to the theoretical values within an error of about $\pm 5.6 \%$. The followings are the resultant

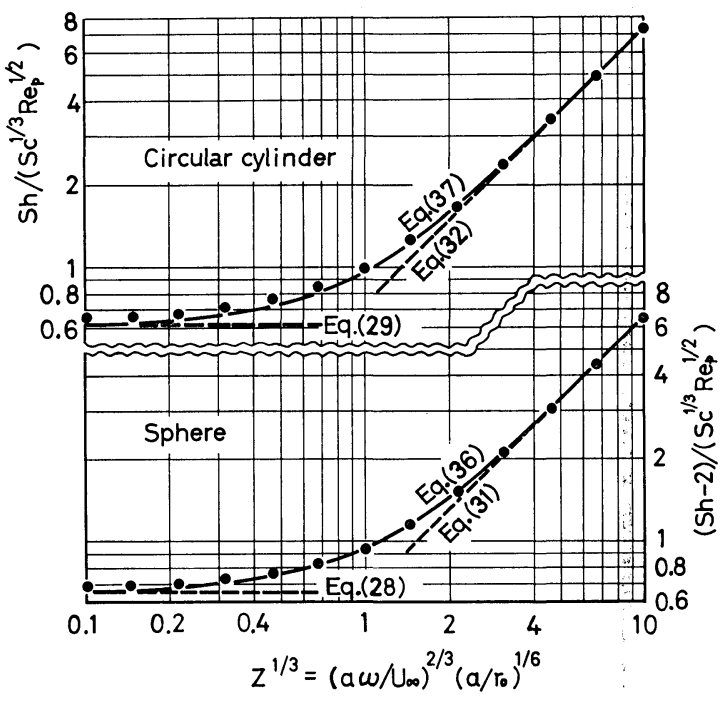

- : theoretical value (numerically calculated from Eqs. (13) to (16) and (24) to (27))

Fig. 2. Overall mass-transfer under pulsating flow.

Table 1. Summary of mass transfer from a circular cylinder in steady flow.

\begin{tabular}{l|clll}
\hline \multicolumn{1}{c|}{ Author } & Year & \multicolumn{1}{c}{ Remarks } & Formulas & Applicable range \\
\hline Friedlander ${ }^{22)}$ & 1957 & Analysis based on boundary-layer theory & $S h=0.557 S c^{1 / 3} R e_{p}^{1 / 3}$ & $R e_{p} \approx 0.1, S c \gg 1$ \\
Kiser \& Hoelscher ${ }^{23)}$ & 1957 & Mass-transfer experiments & $S h=0.211 S c^{1 / 3} R e_{p}^{0.57}$ & $5000<R e_{p}<70000$ \\
Grafton $^{20)}$ & 19.63 & Analysis based on boundary-layer theory & $S h=0.90 S c^{1 / 3} R e_{p}^{1 / 2}$ & $R e_{p}<6 \times 10^{6}, S c \gg 1$ \\
\hline
\end{tabular}

\begin{tabular}{|c|c|c|c|c|c|}
\hline Author & Year & Remarks & $B_{1}$ & $B_{2}$ & Applicable range \\
\hline $\begin{array}{l}\text { Noordsij \& } \\
\text { Rotte }^{4}\end{array}$ & 1967 & $\begin{array}{l}\text { Mass-transfer experiments (vibrating } \\
\text { sphere in a fluid at rest) }\end{array}$ & 2 & 0.135 & $\begin{array}{l}2.5 \leqq f \leqq 35 \mathrm{~Hz}, 500<S c<2000 \\
0.075 \leqq a \leqq 0.1575 \mathrm{~cm}\end{array}$ \\
\hline Mori et al. ${ }^{24)}$ & 1969 & $\begin{array}{l}\text { Analysis and heat- and mass-transfer } \\
\text { experiments (under pulsating flow) }\end{array}$ & 2 & 0.595 & $\begin{array}{l}23 \leqq f \leqq 70 \mathrm{~Hz},\left|u_{o s}\right|<6 \mathrm{~m} / \mathrm{s} \\
U_{\infty}>3 \mathrm{~m} / \mathrm{s}, 0.6<\operatorname{Sc}(\operatorname{Pr})<1.6\end{array}$ \\
\hline Kitaura et $a l .{ }^{3)}$ & 1969 & $\begin{array}{l}\text { Analysis based on boundary-layer } \\
\text { theory and mass-transfer experiments } \\
\text { (vibrating sphere in steady flow) }\end{array}$ & 0 & $0.53\left(a / r_{o}\right)^{1 / 6}$ & $\begin{array}{l}1 \leqq f \leqq 11 \mathrm{~Hz}, 1 \leqq a \leqq 4 \mathrm{~cm} \\
40<R e_{p}<300, S_{c}=2.85\end{array}$ \\
\hline Hara et al..$^{25)}$ & 1971 & $\begin{array}{l}\text { Mass-transfer experiments (under } \\
\text { ultrasonic wave) }\end{array}$ & 0 & $10.1\left(a / r_{o}\right)^{1 / 6}$ & $2 \times 10^{5} \leqq f \leqq 2 \times 10^{6} \mathrm{~Hz}$ \\
\hline
\end{tabular}

Table 2. Summary of mass transfer from a sphere in oscillating flow.

$$
S h=B_{1}+B_{2} S c^{1 / 3}\left(r_{o} a \omega / \nu\right)^{1 / 2}
$$

$\left|u_{o s}\right|$ : magnitude of oscillating velocity. 


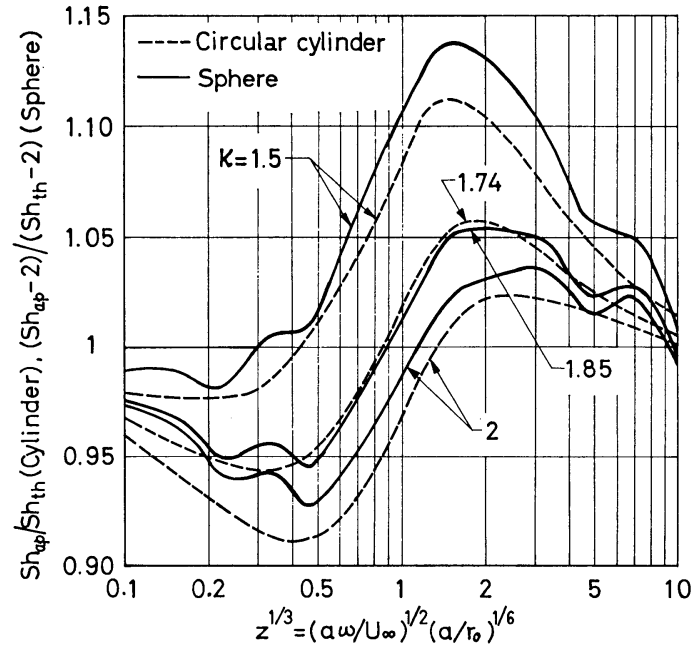

$S h_{a p}$ : Eq. (34) for a sphere and Eq. (35) for a circular cylinder

$S h_{t h}$ : numerically calculated from Eqs. (13) to (16) and (24) to (27)

Fig. 3. Comparisons of approximate expressions for mass transfer under pulsating flow $S h_{a p}$ with theoretical values $S h_{t h}$.

equations (see Fig. 2):

$$
S h=\left\{\begin{array}{r}
2+\left[0.456+\left(0.648 z^{1 / 3}\right)^{1.85}\right]^{1 / 1.85} S c^{1 / 3} R e_{p}^{1 / 2} \\
(\mathrm{~S} .) \quad \ldots \ldots \ldots . .(36) \\
{\left[0.429+\left(0.728 z^{1 / 3}\right)^{1.74}\right]^{1 / 1.74} S c^{1 / 3} R e_{p}^{1 / 2}} \\
\text { (C. C. }) \ldots \ldots .(37)
\end{array}\right.
$$

Up to the present, there have been a lot of reports ${ }^{26,27)}$ which have shown that the turbulence in the main stream accelerates the mass transfer, but only a few on pulsating flow. Mori et al..$^{24)}$ have analyzed heat and mass transfer from a sphere in pulsating flow with large amplitude by theoretical consideration and experiments, and have given the simple correlation equation for oscillating flow, as shown in Table 2, but a complicated equation for pulsating flow, having parameters which have to be evaluated by experiments. In the preceding paper, ${ }^{1}$ the equation of Kitaura et al. ${ }^{3}$ :

$$
S h=\left[\left(2+0.55 S c^{1 / 3} R e_{p}^{1 / 2}\right)^{3}+\left(0.375 z^{1 / 3} S c^{1 / 3} R e_{p}^{1 / 2}\right)^{3}\right]^{1 / 3}
$$

was used to analyze the rate of reduction of single iron oxide pellets by pulsating flow of hydrogen, but it is not clear to be able to use it as it is, because the coefficient 0.375 in this equation was determined by the experiments in which single spheres oscillated in steady flow. Their experimental conditions and the Sherwood number for $z \rightarrow \infty$ are already shown in Table 2. The functional forms of Eqs. (36) and (38) are the same except how to deal with the term " 2 " for the Sherwood number without convection. Therefore, after the results for steady and oscillating flows are taken into account, it is understood that Eq. (36) is satisfactory to some extent, but further examinations by experiments are necessary. ${ }^{13)}$

\section{Conclusions}

Both the local and the overall mass-transfer from a sphere and a circular cylinder in steady, oscillating, and pulsating flows are analyzed. Conclusions obtained are as follows:

(1) Both the local and the overall coefficients of mass-transfer from one of these bodies in steady flow are analytically derived (see Eqs. (11) to (17)) by using previously reported solutions to velocities around these bodies. The resultant correlation equations (see Eqs. (28) and (29)) are much the same as those reported previously. $.20,22,23)$

(2) Both the local and the overall mass-transfer coefficients for a sphere and the local one for a circular cylinder in oscillating flow are analytically derived (see Eqs. (19) to (22), (14) to (16)) by using solutions to velocities around these bodies-solutions derived here from the boundary-layer theory for a sphere (see Appendix) and reported by Schlichting. ${ }^{11)}$ The resultant equation of overall Sherwood number for a sphere (see Eq. (31)) is shown to be rather satisfactory by comparing it with previously reported (semi-)empirical ones. ${ }^{3,4,24,25)}$

(3) From the fact that pulsating flow field is approximately represented by superposing steady flow field upon oscillating flow field, ${ }^{12,13)}$ both the local and the overall coefficients of mass transfer from the bodies are analytically derived (see Eqs. (12) to (16), (24) to (27)). As a result, the overall Sherwood numbers are certain functions of $z=\left(a \omega / U_{\infty}\right)^{3 / 2}\left(a / r_{0}\right)^{1 / 2}$. Hence, correlation equations which approximate the theoretical values calculated numerically from Eqs. (13) to (16) and (24) to (27) are presented (see Eqs. (36) and (37)).

(4) Comparison between local mass-transfer distributions around the bodies in steady and pulsating flows shows that pulsating flow accelerates the mass transfer particularly on the rear side of the bodies, where the mass transfer under steady flow is small because of the dead-water region (see Fig. 1).

\section{Nomenclature}

$a:$ amplitude of oscillation (cm)

$B_{1}, B_{2}:$ constant and coefficient [see Eq. (33)]

$C, C_{0}$ : concentration of gas, and concentration of gas at the surface of a body $\left(\mathrm{g} / \mathrm{cm}^{3}\right)$

$D:$ diffusion coefficient $\left(\mathrm{cm}^{2} / \mathrm{s}\right)$

$k_{f}, k_{f \theta}$ : overall and local mass-transfer coefficients $(\mathrm{cm} / \mathrm{s})$

$R e_{p}:$ Reynolds number associated with pellet (sphere or circular cylinder) $\equiv 2 r_{o} U_{\infty} / \nu$

$r:$ radius of the cross section of a sphere $(\mathrm{cm})$

$r_{o}:$ radius of sphere or circular cylinder $(\mathrm{cm})$

Sc: Schmidt number $\equiv \nu / D$

$S h:$ overall Sherwood number $\equiv 2 r_{o} k_{f} / D$

$S h_{\theta}:$ local Sherwood number $\equiv 2 r_{o} k_{f \theta} / D$

$t:$ time (s)

$U:$ potential flow velocity $(\mathrm{cm} / \mathrm{s})$

$U_{o}$ : amplitude of potential flow velocity $(\mathrm{cm} / \mathrm{s})$

$U_{\infty}:$ steady free-stream-velocity $(\mathrm{cm} / \mathrm{s})$

$u, v$ : velocity components in the directions of $x$ and $y(\mathrm{~cm} / \mathrm{s})$ 
$x, y: \quad$ curvilinear co-ordinates parallel and perpendicular to the wall of a body $(\mathrm{cm})$

$z \equiv\left(a \omega / U_{\infty}\right)^{3 / 2}\left(a / r_{o}\right)^{1 / 2}$

$\beta$ : defined by Eq. (4) $\left(\mathrm{s}^{-1}\right)$

$\Gamma:$ Gamma function $[\Gamma(1 / 3)=2.68]$

$\Delta$ : parameter defined by Eq. (8) or (8-a) (cm) $\zeta_{1}, \zeta_{2^{n}}$ : functions of $\eta$, defined by Eqs. (A-11) and (A-15), respectively

$\eta \equiv y \sqrt{\omega / \nu}$

$\theta:$ angle in spherical or cylindrical co-ordinates $\equiv x / r_{o}(\mathrm{rad}, \mathrm{deg})$

$\theta_{s}: \quad$ angle of separation point ( $\left.\mathrm{rad}, \mathrm{deg}\right)$

$\kappa$ : exponent (see Eqs. (34) and (35))

$\nu$ : kinematic viscosity of gas $\left(\mathrm{cm}^{2} / \mathrm{s}\right)$

$\phi:$ variable defined by Eq. (7) $\left(\mathrm{s} / \mathrm{cm}^{2}\right)$

$\phi$ : stream function $\left(\mathrm{cm}^{3} / \mathrm{s}\right.$ for a sphere, or $\mathrm{cm}^{2} / \mathrm{s}$ for a circular cylinder)

$\omega:$ angular frequency $(\mathrm{rad} / \mathrm{s})$

\section{Subscript}

ta: time-averaged quantities

\section{Acknowledgements}

The authors wish to express their thanks to Mr. Y. Masuyama (formerly student of Osaka University, now engineer of Kubota, Ltd.) for his assistance.

\section{REFERENCES}

1) M. Ohmi and T. Usui: Tetsu-to-Hagané, 59 (1973), 1902; Trans. ISIJ, 16 (1976), 85.

2) M. Ohmi and T. Usui: Tetsu-to-Hagané, 59 (1973), 1888; Trans. ISIJ, 16 (1976), 77.

3) Y. Kitaura, H. Tanaka, G. Ueda and N. Kojima: Kagaku Kögaku (Chemical Eng. Japan), 33 (1969), 175.

4) P. Noordsij and J. W. Rotte: Chem. Eng. Sci., 22 (1967), 1475.

5) G. J. Jameson: Chem. Eng. Sci., 19 (1964), 793.

6) Y. Sugano and D. A. Ratkowsky: Chem. Eng. Sci., 23 (1968), 707.

7) J. F. Davidson and E. J. Cullen: Trans. Inst. Chem. Eng., 35 (1957), 51.

8) H. Schlichting: Boundary-layer Theory, McGraw-Hill Book Co., New York, (1968), 154, 158, 226, 298 \& 391.

9) F. W. Scholkemeier: Arch. Math., 1 (1948/1949), 270.

10) M. J. Lighthill: Proc. Roy. Soc. Lond., Ser. A, 224 (1954), 1.

11) H. Schlichting: Phys. Z., 33 (1932), 327.

12) M. Ohmi, T. Usui, M. Fukawa and S. Hirasaki: Trans. Japan Soc. Mech. Engrs., 41 (1975), 2043; Bull. JSME, 19 (1976), 298.

13) M. Ohmi, T. Usui, Y. Matsumoto and Y. Masuyama: Tetsu-to-Hagané, 64 (1978), 1105; Trans. ISIJ, 22 (1982), 600.

14) R. B. Bird, W. E. Stewart and E. N. Lightfoot: Transport Phenomena, John Wiley \& Sons, New York, (1960), 303.

15) N. Frössling: Gerl. Beitr. Geophys., 52 (1938), 170.

16) M. Linton and K. L. Sutherland: Chem. Eng. Sci., 12 (1960), 214.

17) H. Gibert and H. Angelino: Chem. Eng. Sci., 28 (1973), 855.

18) J. S. Lewis: Int'l. J. Heat Mass Transfer, 14 (1971), 325.

19) M. Lebouche and M. Martin: Int'l. J. Heat Mass Transfer, 18 (1975), 1161.

20) R. W. Grafton: Chem. Eng. Sci., 18 (1963), 457.

21) F. Ihme, H. Schmidt-Traub and H. Brauer: VDI-Be- richte, No. 187, (1972), 84.

22) S. K. Friedlander: AIChEJ, 3 (1957), 43.

23) K. M. Kiser and H. E. Hoelscher: Indust. Eng. Chem., 49 (1957), 970.

24) Y. Mori, M. Imabayashi, K. Hijikata and Y. Yoshida: Int'l. J. Heat Mass Transfer, 12 (1969), 571.

25) H. Hara, K. Shimada and K. Endoh: Kagaku Kōgaku, 35 (1971), 597.

26) Y. Kitaura and K. Aoki: Kagaku Kögaku, 24 (1960), 134.

27) K. Endoh, H. Tsuruga, H. Hirano and M. Morihira: Kagaku Kögaku, 35 (1971), 257.

28) K. Endoh and H. Hirano: Bull. Fac. Eng. of Hokkaido Univ., 60 (1971), 111.

\section{Appendix}

For unsteady viscous incompressible flow around a sphere, combination of equations of motion inside and outside the boundary layer ${ }^{8)}$ yields

$$
\frac{\partial u}{\partial t}+u \frac{\partial u}{\partial x}+v \frac{\partial u}{\partial y}=\frac{\partial U}{\partial t}+U \frac{\partial U}{\partial x}+\nu \frac{\partial^{2} u}{\partial y^{2}}, \ldots
$$

where,

$$
\begin{aligned}
& U(x, t)=U_{o}(x) e^{i \omega t}, \\
& U_{o}(x)=(3 / 2) a \omega \sin \left(x / r_{o}\right) \text {, } \\
& u=(1 / r) \partial \phi / \partial y, \quad v=-(1 / r) \partial \phi / \partial x \text {, }
\end{aligned}
$$

and $\quad r(x)=r_{o} \sin \left(x / r_{o}\right)$

Equation (A-2) with Eq. (A-3) gives the potential flow around the sphere oscillating harmonically with angular frequency $\omega$ and amplitude $a$ in a stagnant fluid; the co-ordinates are fixed in the body.

For a small amplitude oscillation, Eq. (A-1) can be solved analytically, if the following approximate relations are used:

$$
\begin{aligned}
& \left.\begin{array}{l}
u(x, y, t)=u_{1}(x, y, t)+u_{2}(x, y, t), \\
v(x, y, t)=v_{1}(x, y, t)+v_{2}(x, y, t),
\end{array}\right\} . . \\
& \partial u_{1} / \partial t-\nu \partial^{2} u_{1} / \partial y^{2}=\partial U / \partial t, \ldots \ldots \ldots \ldots \\
& \frac{\partial u_{2}}{\partial t}-\nu \frac{\partial^{2} u_{2}}{\partial y^{2}}=U \frac{\partial U}{\partial x}-u_{1} \frac{\partial u_{1}}{\partial x}-v_{1} \frac{\partial u_{1}}{\partial y}
\end{aligned}
$$

The boundary conditions are

$$
\begin{aligned}
& y=0: u_{1}=v_{1}=0 ; \quad y=\infty: u_{1}=U, \ldots \ldots \ldots \ldots(\mathrm{A} \\
& y=0: u_{2}=v_{2}=0 ; \quad y=\infty: u_{2, o s}=0, u_{2, t a}=\text { finite. }
\end{aligned}
$$

The first approximation to the stream function is assumed as

$$
\phi_{1}(x, y, t)=\sqrt{\nu / \omega} U_{o} r \zeta_{1}(\eta) e^{i \omega t},
$$

where,

$$
\eta=y \sqrt{\omega / \nu} \text {. }
$$

Insertion of Eq. (A-11) into Eq. (A-4) yields

and

$$
\begin{aligned}
& u_{1}=U_{o} \zeta_{1}^{\prime} e^{i \omega t}, \ldots \ldots \ldots \ldots \ldots \ldots \ldots \ldots \ldots \ldots \ldots \\
& v_{1}=-\sqrt{\frac{\nu}{\omega}}\left\{\frac{d U_{o}}{d x}+\frac{U_{o}}{r} \frac{d r}{d x}\right\} \zeta_{1} e^{i \omega t},
\end{aligned}
$$

where the prime denotes differentiation with respect to $\eta$. It follows from Eqs. (A-2), (A-7), (A-9), and (A-12) that

$$
\zeta_{1}=-\frac{1-i}{\sqrt{2}}+\eta+\frac{1-i}{\sqrt{2}} e^{-(1+i) \eta / \sqrt{2}} .
$$


The second approximation to the stream function is assumed as follows:

$$
\begin{aligned}
\phi_{2}(x, y, t)= & \sqrt{\frac{\nu}{\omega}} U_{o} \frac{d U_{o}}{d x} \frac{r}{\omega}\left\{\zeta_{2 a}(\eta) e^{2 i \omega t}+\zeta_{2 b}(\eta)\right\} \\
& +\sqrt{\frac{\nu}{\omega}} \frac{U_{o}^{2}}{\omega} \frac{d r}{d x}\left\{\zeta_{2 c}(\eta) e^{2 i \omega t}+\zeta_{2 d}(\eta)\right\} .
\end{aligned}
$$

Insertion of Eq. (A-15) into Eq. (A-4) yields

$$
u_{2}=\frac{U_{o}}{\omega} \frac{d U_{o}}{d x}\left(\zeta_{2 a}^{\prime} e^{2 i \omega t}+\zeta_{2 b}^{\prime}\right)+\frac{U_{o}^{2}}{\omega r} \frac{d r}{d x}\left(\zeta_{2 c}^{\prime} e^{2 i \omega t}+\zeta_{2 d}^{\prime}\right)
$$

and an equation for $v_{2}$. By inserting the real parts of $U, u_{1}, v_{1}$, and $u_{2}$ into Eq. (A-8), the following equations are obtained:

$$
\begin{aligned}
& 2 i \zeta_{2 a}^{\prime}-\zeta_{2 a}^{\prime \prime \prime}=\left(1-\zeta_{1}^{\prime 2}+\zeta_{1} \zeta_{1}^{\prime \prime}\right) / 2, \ldots \ldots . . \\
& \zeta_{2 b}^{\prime \prime \prime}=-1 / 2+\zeta_{1}^{\prime} \zeta_{1}^{\prime} / 2-\left(\zeta_{1} \bar{\zeta}_{1}^{\prime \prime}+\bar{\zeta}_{1} \zeta_{1}^{\prime \prime}\right) / 4, \\
& 2 i \zeta_{2 c}^{\prime}-\zeta_{2 c}^{\prime \prime \prime}=\zeta_{1} \zeta_{1}^{\prime \prime} / 2, \ldots \ldots \ldots \ldots \ldots \ldots \ldots \ldots . . . \ldots \ldots \ldots \ldots \ldots
\end{aligned}
$$

and $\quad \zeta_{2 d}^{\prime \prime \prime}=-\left(\zeta_{1} \bar{\zeta}_{1}^{\prime \prime}+\bar{\zeta}_{1} \zeta_{1}^{\prime \prime}\right) / 4$,

where the bar over the symbols denotes the conjugate complex quantities. The boundary conditions (A10) are rewritten as

$$
\left.\begin{array}{l}
\eta=0: \quad \zeta_{2 n}=\zeta_{2 n}^{\prime}=0 \quad(n=a, b, c, d) ; \\
\eta=\infty: \zeta_{2 a}^{\prime}=\zeta_{2 c}^{\prime}=0, \quad \zeta_{2 b}^{\prime}=\text { finite }, \zeta_{2 d}^{\prime}=\text { finite. }
\end{array}\right\}
$$

Thus,

$$
\begin{aligned}
\zeta_{2 a}= & \frac{1+i}{4} e^{-(1+i) \eta}+\frac{i}{2} \eta e^{-(1+i) \eta / \sqrt{2}}-\frac{1+i}{4}, \ldots( \\
\zeta_{2 b}= & -\frac{1}{4 \sqrt{2}} e^{-\sqrt{2} \eta}-\frac{3}{\sqrt{2}} e^{-\eta / \sqrt{2}} \cos \frac{\eta}{\sqrt{2}} \\
& -\sqrt{2} e^{-\eta / \sqrt{2}} \sin \frac{\eta}{\sqrt{2}}-\frac{\eta}{2} e^{-\eta / \sqrt{2}} \sin \frac{\eta}{\sqrt{2}} \\
& -\frac{3}{4} \eta+\frac{13}{4 \sqrt{2}}, \ldots \ldots \ldots \ldots \ldots \ldots \ldots . . . \\
\zeta_{2 c}= & -\frac{1+i}{8 \sqrt{2}} e^{-\sqrt{2}(1+i) \eta}+\frac{7}{8}(1+i) e^{-(1+i) \eta} \\
& -\frac{1+i}{\sqrt{2}} e^{-(1+i) \eta / \sqrt{2}}+\frac{i}{2} \eta e^{-(1+i) \eta / \sqrt{2}}
\end{aligned}
$$

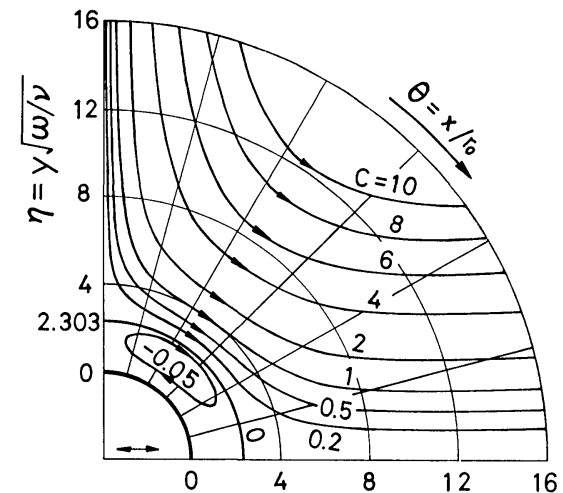

Fig. A-1. Pattern of streamlines of the steady secondary motion around an oscillating sphere.

$$
\begin{aligned}
C & =\phi_{t a} /\left(a^{2} \sqrt{\nu \omega}\right) \\
& =(9 / 4)\left[\zeta_{2 b}(\eta)+\zeta_{2 d}(\eta)\right] \sin ^{2} \theta \cos \theta
\end{aligned}
$$

$$
-\frac{(7 \sqrt{2}-9)(1+i)}{8 \sqrt{2}}
$$

and

$$
\begin{aligned}
\zeta_{2 d}= & -\sqrt{2} e^{-\eta / \sqrt{2}} \cos \frac{\eta}{\sqrt{2}}-\frac{1}{\sqrt{2}} e^{-\eta / \sqrt{2}} \sin \frac{\eta}{\sqrt{ } 2} \\
& -\frac{\eta}{2} e^{-\eta / \sqrt{2}} \sin \frac{\eta}{\sqrt{2}}-\frac{\eta}{2}+\sqrt{ } 2 . \quad \ldots \ldots \ldots .(\mathrm{A}
\end{aligned}
$$

From this second-approximate solution, the steady secondary flow is obtained as follows:

$$
\begin{aligned}
\left(u_{t a}\right)_{y=\infty} & =\left(u_{2, t a}\right)_{y=\infty} \\
& =\frac{U_{0}}{\omega} \frac{d U_{o}}{d x}\left(\zeta_{2 b}^{\prime}\right)_{\eta=\infty}+\frac{U_{o}^{2}}{\omega r} \frac{d r}{d x}\left(\zeta_{2 d}^{\prime}\right)_{\eta=\infty},
\end{aligned}
$$

where, $\quad\left(\zeta_{2 b}^{\prime}\right)_{\eta=\infty}=-3 / 4, \quad\left(\zeta_{2 d}^{\prime}\right)_{\eta=\infty}=-1 / 2$.

From Eqs. (A-3), (A-5), and (A-15), the time-averaged value of the stream function is given as

$$
\psi_{t a}=(9 / 4) a^{2} \sqrt{\nu \omega}\left(\zeta_{2 b}+\zeta_{2 d}\right) \sin ^{2} \theta \cos \theta .
$$

As is shown in Fig. A-1, the flow is symmetrical about $\theta=\pi / 2$, but not about $\theta=\pi / 4$; the flow for a circular cylinder ${ }^{11)}$ is symmetrical with both $\theta=\pi / 2$ and $\pi / 4$. Endoh and Hirano ${ }^{28)}$ have observed such a steady secondary flow around a sphere. 